\title{
Low-Power Adaptive Pseudo Noise Code Acquisition for Spread-Spectrum Systems
}

\author{
Mau-Lin Wu and Kuei-Ann Wen
}

\begin{abstract}
A novel pseudo noise code acquisition combined with the newly proposed adaptive sampling rate and threshold control (ASTC) algorithm is derived for low-power spread-spectrum systems with complementary metal-oxide-semiconductor implementations. Low-power performance can be achieved by reducing the sampling rate of the proposed system while maintaining the system performance. The sampling rate is dynamically updated due to the change of the channel noise level. Under the assumptions that the channel is additive white Gaussian noise slow-fading channel, up to $\mathbf{7 4 . 3 \%}$ reduction in power consumption compared to the conventional fixed-sampling rate and fixed-threshold architecture is demonstrated with insignificant increase of system complexity. The proposed architecture can be applied to the design of low-power and controllable-performance spread-spectrum communication systems.
\end{abstract}

Index Terms-Adaptive computing, code acquisition, communication, low-power design, spread spectrum.

\section{INTRODUCTION}

I NCREASING the system capacity for wireless communication systems is required due to the explosion of wireless applications such as data and multimedia. According to the requirements for large system capacity and interference rejection immunity, code-division multiple-access (CDMA) becomes one of the most popular candidates for modern wireless communication systems. The spread-spectrum technique plays a vital role in the CDMA system and the pseudo noise (PN) code acquisition system is the major module for receiver performance.

Among the design parameters of the mobile wireless communication system, power consumption is one of the major concerns. In this paper, we propose a novel low-power code acquisition structure applied to spread-spectrum systems. The design is targeted for complementary metal-oxide-semiconductor (CMOS) implementation to be applied to fully silicon wireless terminals.

It is well-known that the power consumption in a CMOS very large scale integration (VLSI) system is dominated by the dynamic power. Therefore, low-power designs are mostly focused on reducing the dynamic power consumption. The dynamic power consumption $P_{d}$ of CMOS VLSI can be modeled

Manuscript received August 18, 2000; revised July 10, 2001; accepted August 3,2001 . The editor coordinating the review of this paper and approving it for publication is C. Nicol. This work was supported in part by the Ministry of Education and in part by the National Science Council, R.O.C. under Contract 89-E-FA06-2-4.

M.-L. Wu is with Logic Research and Development Department I, Integrated Circuit Solution Inc., Hsin-Chu, Taiwan (e-mail: daniel_wu@icsi.com.tw).

K.-A. Wen is with the Institute of Electronics Engineering, National Chiao

Tung University, Hsin-Chu, Taiwan, R.O.C. (e-mail: kawen@cc.nctu.edu.tw). Digital Object Identifier 10.1109/TWC.2002.804187

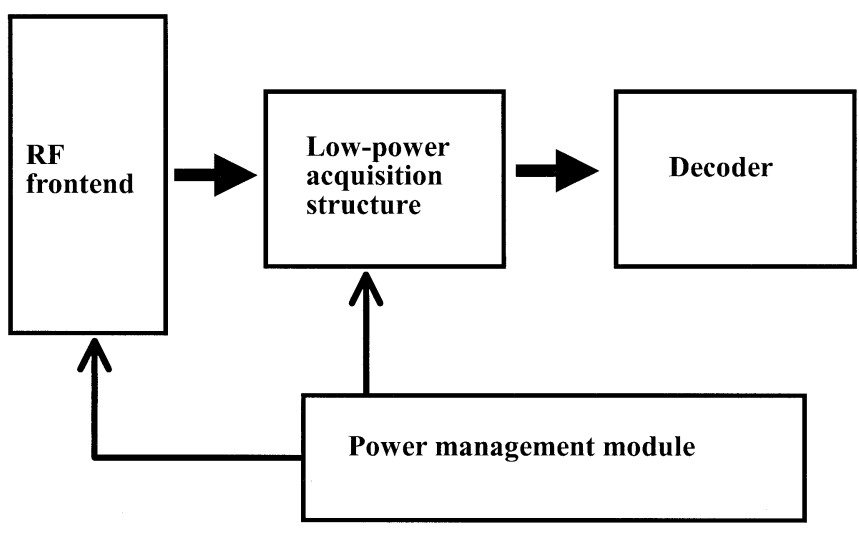

Fig. 1. Functional block diagrams of the receiver of the spread-spectrum communication system.

by the relationship with switched capacitance $C_{L}$, power-supply voltage $V_{D D}$ and switching frequency $f_{\mathrm{sw}}$ by the following [1]:

$$
P_{d}=C_{L} V_{D D}^{2} f_{\mathrm{sw}}
$$

The dynamic power consumption of a CMOS VLSI system could be reduced by reducing either switching frequency [2]-[7], power-supply voltage [8]-[14], or switched capacitance [15]-[18]. In this paper, the low-power system is derived by reducing the switching frequency.

For the low-power architectures of the PN code acquisition system, two different approaches were proposed in [19] and [20]. The architecture in [19] combines the matched filters and serial correlators to achieve low-power application. The parallel spread-spectrum correlator was applied in [20] to reduce the power consumption of the code acquisition system. Research about adaptation of threshold by estimating power level value to maintain system performance was proposed in [21]. Multiplying the estimated background power level with threshold coefficient is an implementation with simple system complexity.

This paper proposes an adaptive PN code acquisition system and achieves low-power operation by dynamically reducing the sampling rate and updating the threshold according to varying channel noise. System performance is maintained above a desired specification. The sampling rate and the threshold of the proposed architecture will be dynamically updated due to changes in the channel noise level. The adaptation engine for sampling rate and threshold to the channel noise level can be implemented either real-time or off-line by power management modules. The system architecture is illustrated in Fig. 1. Detailed functional blocks in the proposed system will be disclosed in the next section. 


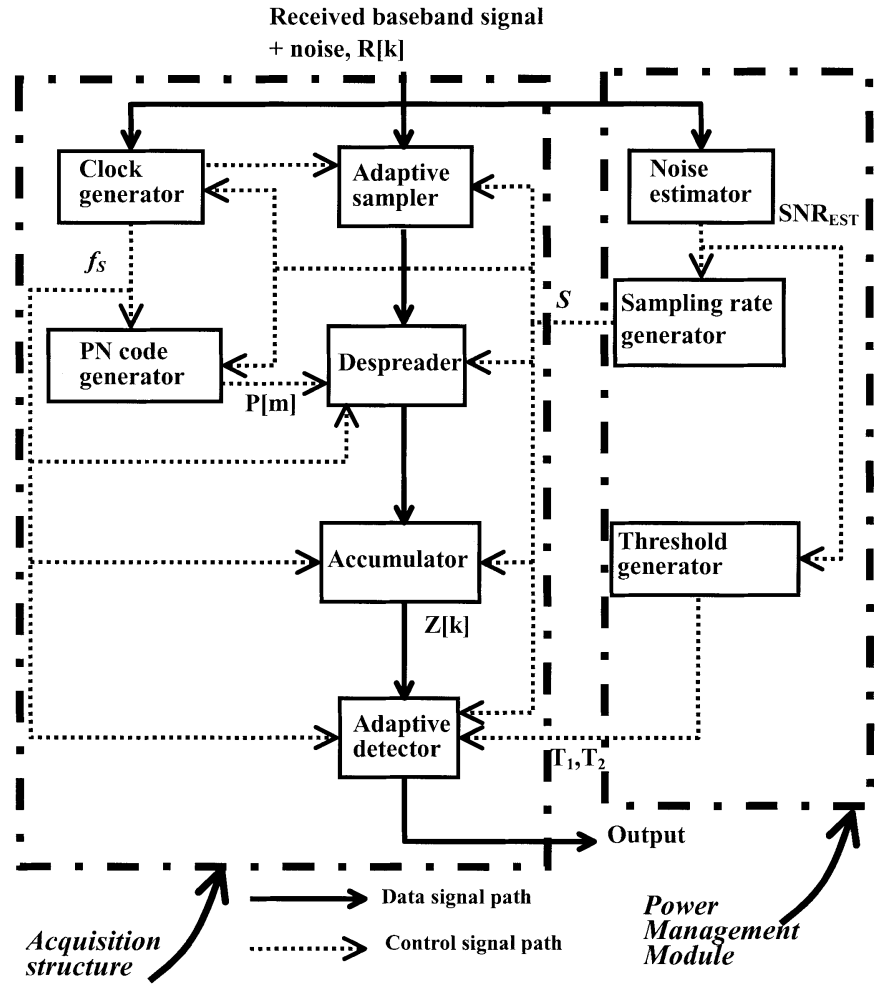

Fig. 2. Functional blocks of the proposed low-power code acquisition structures.

The low-power adaptive code acquisition system is described in Section II. In Section III, we analyze the power consumption and system performance. We propose a novel adaptive sampling rate and threshold control algorithm in Section IV. Simulations of system performance are demonstrated in Section V. Comparisons of the power consumption and system performances are also listed. The expansion and limitation about the proposed architecture are discussed in Section VI. Finally, conclusions were made in Section VII.

\section{LOW-POWER AdAPTIVE CODE ACQUISITION SySTEM}

\section{A. System Architecture of the Proposed Code Acquisition System}

Functional blocks of the proposed low-power code acquisition structure are shown in Fig. 2. The received baseband signal added with the channel noise is the input signal of this code acquisition structure. The output is the decision of the "adaptive detector." The "adaptive sampler" adjusts the sampling tone according to the sampling rate generator. The output data of this adaptive sampler is then despread by the PN sequence in the block of "despreader." The output data of the despreader is then accumulated for the period of the PN sequence. After that, the "adaptive detector" makes the detection result according to the output data of the accumulator. The decision threshold in the block of adaptive detector is adapted according to the threshold generator to optimize the system performance.

The received baseband signal is also processed to extract the clock signal, which is required to generate the local PN sequence. The function of the "noise estimator" is to estimate the channel noise level from the received baseband signal. The noise estimator can be implemented by several well-known techniques [22]-[24]. The operating frequency of the proposed acquisition structure is the sampling rate $S$, which is generated by the sampling rate generator according to the estimated channel noise level $\mathrm{SNR}_{\mathrm{EST}}$. The thresholds $T_{1}$ and $T_{2}$ are generated by a threshold generator according to the estimated

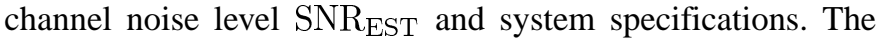
adaptive detector makes the decision based upon these two threshold values. Because the operating frequency of this structure is adaptive, the "clock generator" is designed to be variable. Accordingly, the "PN code generator" is designed to generate the $\mathrm{PN}$ sequence with a varying sampling rate.

For the conventional PN code acquisition system with the fixed sampling rate and the fixed decision thresholds, there always exists the trade off between power consumption and system performance. For example, the system performance of one conventional PN code acquisition system can be improved by increasing the sampling rate, but the power consumption is also increased accordingly. However, the power consumption and the system performance are considered together in the proposed architecture.

\section{B. System Complexity}

The proposed system contains two blocks, the acquisition structure, and the power management module. Two modules make up the acquisition structure as shown in Fig. 2. They are the adaptive sampler and adaptive detector blocks. The varying sampling rate of the adaptive sampler is implemented by varying the system clock with no extra circuitry needed. The adaptive detector is implemented with adjusted threshold and, thus, there is actually no hardware required. The acquisition structure is, therefore, implemented without hardware support.

The power management module can be easily implemented by a processor or digital signal processor (DSP) module. [25]-[27] The sampling rate and threshold generators are realized using a lookup table with the input being the estimated channel noise level and the outputs are sampling rate and threshold value, respectively. Therefore, the adaptation can be implemented with simple memory access instructions. With minor hardware and software additions, the adaptive sampler and detector can be implemented to achieve significant performance improvement.

\section{AdAPTATION ANALYSIS OF POWER CONSUMPTION AND SYSTEM PERFORMANCE}

In this section, the analysis of power consumption and system performance of the proposed structure is discussed according to the adaptive scheme of sampling rate and decision threshold.

\section{A. System Performance Analysis}

Considering the system performance of the PN code acquisition system, the average acquisition time is most important. However, the average acquisition time depends on the system structure and the probability of detection and false alarm [28]. The average acquisition time decreases when the probability of detection is increasing or the probability of false alarm is decreasing. The PN code acquisition system with the smaller 


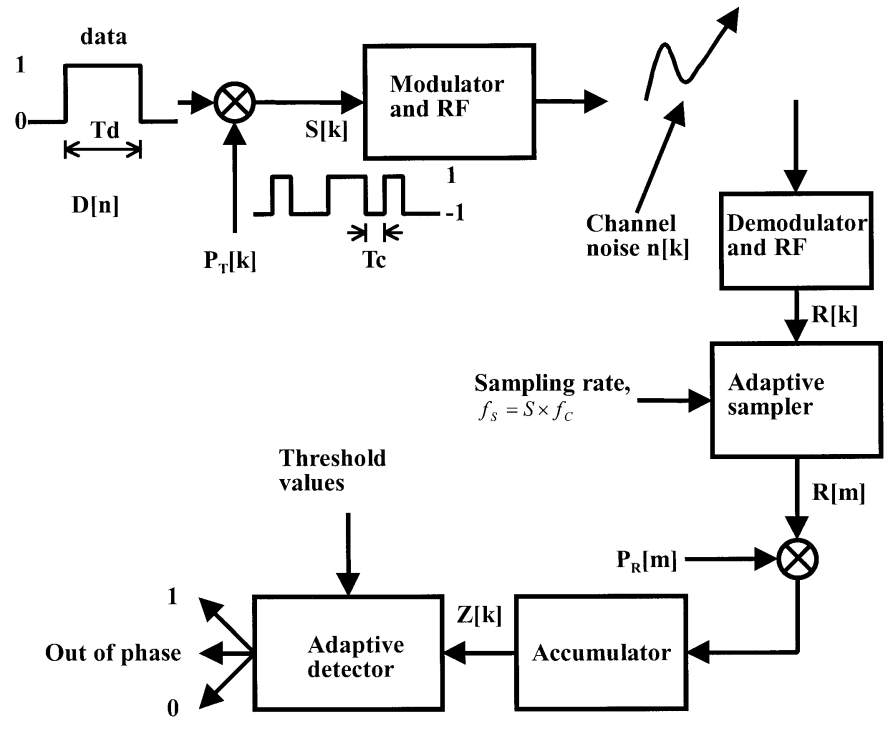

Fig. 3. Functional block diagrams of the proposed direct sequence spread-spectrum communication system.

average acquisition time has better performance than that with larger average acquisition time. Therefore, the probabilities of false alarm and detection are two important specifications for the PN code acquisition system. To improve the system performance, the probability of detection should be increased and the probability of false alarm should be decreased. In the following, the probabilities of detection and false alarm of the proposed system are analyzed as the functions of channel noise level and sampling rate.

The functional block diagrams of the proposed direct sequence spread-spectrum (DSSS) communication system are shown in Fig. 3. The source data $D[n]$ is with the data rate $1 / T_{d}=f_{d}$ and in the format of 0 and 1 . Before the source data is modulated and transmitted, it will be multiplexed by the PN sequence $P_{T}[k] . P_{T}[k]$ is the PN sequence with code length $N$ and chip rate $f_{c}$, which is applied in this DSSS communication system. The relationship between the data rate $f_{d}$ and the chip rate $f_{c}$ is $f_{c}=1 / T_{c}=N \times f_{d}$, where $N$ is called the processing gain of the system. In general, $N$ is equal to the length of the PN code sequence. $S[k]$ is the spreaded data with the chip rate $f_{c}$, which is ready for modulation and transmitting. The relationship between $S[k]$ and $D[n]$ can be modeled as

$$
S[k]=P_{T}\left[((k))_{N}\right] \times D\left[\left\|\frac{k}{N}\right\|\right]
$$

where $((k))_{N}$ is the remainder of $k$ divided by $N$ and $\|x\|$ is the largest integer less than or equal to $x$. The value of $S[k]$ is either 1 or -1 . After demodulation, the received signal is converted to $R[k]$ through the matched filter. With channel, demodulation and RF front end being modeled with additive white Gaussian noise (AWGN) $n[k]$ and phase noise $\tau$, the received spreaded signal $R[k]$ can be modeled as the transmitted signal $S[k]$ plus the channel noise $n[k]$, which is shown

$$
R[k]=S[k]+n[k] .
$$

The phase noise introduced can be modeled in the despreading procedure. The received signal at the receiver $R[k]$ is sampled
TABLE I

HYPOTHESIS OF $H_{1}, H_{0}, G_{1}$, AND $G_{0}$

\begin{tabular}{c|l}
\hline $\begin{array}{c}\text { Hypothes } \\
\text { is }\end{array}$ & \\
\hline$H_{1}$ & Signal ' 1 ' is transmitted. \\
\hline$H_{0}$ & Signal ' 0 ' is transmitted. \\
\hline$G_{1}$ & The phase of PN code is synchronous with received data. \\
\hline$G_{0}$ & The phase of PN code is not synchronous with received data. \\
\hline
\end{tabular}

at the rate of $f_{s}$. The sampling rate is correlated to the chip rate at the relationship shown

$$
f_{S}=S \times f_{C} .
$$

That is to say, there are $S$ samples in each period of the chip of the received signal. The sampled signal $R[m]$ is then despreaded by the local generated PN sequence, $P_{R}[m+\tau]$ with the phase difference $\tau$ compared to $R[m]$. If $\tau=0$, the phase of $R[m]$ and $P_{R}[m+\tau]$ is the same and the absolute of the output value of the accumulator should be larger than the threshold of the detector. In this condition, data is recognized. Otherwise, with the output value of the accumulator being smaller than the threshold, next chip will be checked until the correct phase is detected.

The output value of the accumulator is termed as " $Z[k]$ " and the thresholds of the detector are $T_{1}$ and $T_{2}$. If $Z[k]>T_{1}$, then the system detects the " 1 " signal. If $Z[k]<T_{2}$, then the system detects the " 0 " signal. If the phase of the PN sequence is not synchronized with that of the received signal $R[m]$, the phase of the received signal is not locked and no output values are decided at the detector. In the following, we want to derive the expressions for the probability distributions of detection and false alarm of the proposed system. Let us denote the hypothesis that signal " 1 " is transmitted by $H_{1}$. The hypothesis that signal " 0 " is transmitted is termed by $H_{0}$. On the other hand, we also denote the hypothesis that the phase of the local-generated PN sequence is synchronous with that of the received signal by $G_{1}$ and it is denoted by $G_{0}$ if the sequence is not synchronous. Table I summarizes these hypothesis.

There are two possible events for probability of detection, which is denoted by $P_{D}$. One event is that the receiver detects the "1" signal and the phase is synchronous between local PN sequence and received signal when " 1 " signal is transmitted. Another event is that the receiver detects the " 0 " signal and the phase is synchronous between local PN sequence and received signal when " 0 " signal is transmitted. Therefore, the probability of detection can be expressed as

$$
P_{D}=\operatorname{Pr}\left\{Z>T_{1} \mid H_{1} \cap G_{1}\right\}+\operatorname{Pr}\left\{Z<T_{2} \mid H_{0} \cap G_{1}\right\} .
$$

The probability of false alarm, which is denoted by $P_{F}$, is the probability that the receiver detects " 1 " or " 0 " signal when the phase is not synchronous. The expression for $P_{F}$ is shown as

$$
P_{F}=\operatorname{Pr}\left\{Z>T_{1} \mid G_{0}\right\}+\operatorname{Pr}\left\{Z<T_{2} \mid G_{0}\right\} .
$$

According to the expressions of $P_{D}$ and $P_{F}$ in (5) and (6), respectively, we can derive $P_{D}$ and $P_{F}$ for the proposed PN code acquisition receiver system. Let us assume that the PN code used in the DSSS communication system is $P_{R}[m]$ with code 
length $N$. Because $P_{R}[m]$ is generated by over-sampling $P_{T}[k]$ by $S$ times. Therefore, if $P_{T}[k]=\left[P_{0}, P_{1}, \ldots, P_{N-1}\right]$, then the expression of $P_{R}[m]$ is shown as

$$
P_{R}[m]=[\underbrace{P_{0}, \ldots, P_{0}}_{S}, \underbrace{P_{1}, \ldots, P_{1}}_{S}, \ldots, \underbrace{P_{N-1}, \ldots, P_{N-1}}_{S}]
$$

where $m=0, \ldots, S N-1$ and $P_{k}= \pm 1, k=0,1, \ldots, N-1$. As described in Fig. 3, the output value of the accumulator can be expressed as

$$
\begin{aligned}
Z[k] & =\frac{1}{S} \sum_{l=-S N+1}^{0} R[k+l] P[k+l+\tau] \\
& =\frac{1}{S} \sum_{l=-S N+1}^{0}(S[k+l]+n[k+l]) P[k+l+\tau]
\end{aligned}
$$

where $\tau=0, S, \ldots, S(N-1)]$. In the expression of $Z[k]$, $n[k+l]$ is the channel noise with the zero mean and variance $\sigma^{2}$. In the following, we will derive the expressions for $P_{D}$ and $P_{F}$.

If $G_{1}$ is true, then $\tau=0$. If $H_{1}$ is true, then $S[k+l]=P[k+l]$. Therefore, $Z[k]$ can be reduced to (9) in this condition

$$
Z[k]=N+\frac{1}{S} \sum_{l=-S N+1}^{0} P[k+l] n[k+l] .
$$

Because $n[k+l]$ are the identical independent Gaussian distributions with mean zero and variance $\sigma^{2}$ and $P[k+l]= \pm 1$, which are constant values, $(1 / S) \sum_{l=-S N+1}^{0}$ $P[k+l] n[k+l]$ is the Gaussian distribution with mean $(1 / S) \sum_{l=-S N+1}^{0} P[k+l] \times 0=0$ and variance $\left(1 / S^{2}\right) \sum_{l=-S N+1}^{0} P[k+l]^{2} \sigma^{2}=N \sigma^{2} / S$. Therefore, $Z[k]$ is the Gaussian distribution with mean $N$ and variance $N \sigma^{2} / S$, which is denoted by $G\left(N, N \sigma^{2} / S\right)$. By the similar derivation, $Z[k]$ is the $G\left(-N, N \sigma^{2} / S\right)$ if $H_{0}$ and $G_{1}$ are true. Let us assume that $\operatorname{Pr}\left\{H_{1}\right\}=\operatorname{Pr}\left\{H_{0}\right\}=1 / 2$. Therefore, the probability of detection $P_{D}$ can be expressed as

$$
P_{D}=\frac{1}{2}\left[1-\frac{1}{2} \operatorname{erf}\left(\frac{T_{1}-N}{\sqrt{\frac{2 N}{S \sigma}}}\right)+\frac{1}{2} \operatorname{erf}\left(\frac{T_{2}+N}{\sqrt{\frac{2 N}{S \sigma}}}\right)\right]
$$

where $\operatorname{erf}(x)$ is the error function, which is defined as

$$
\operatorname{erf}(x)=\frac{2}{\sqrt{\pi}} \int_{0}^{x} e^{-t^{2}} d t .
$$

If $G_{0}$ is true, $P_{F}$ can be derived as

$$
P_{F}=\operatorname{Pr}\left\{Z[k]>T_{1} \mid G_{0}\right\}+\operatorname{Pr}\left\{Z[k]<T_{2} \mid G_{0}\right\}
$$

where $Z[k]$ is defined as (8) for $\tau \neq 0$. In this condition, the probability distribution of $Z[k]$ is a normal distribution with mean $\mu_{F}^{[k, \tau]}$ and variance $N \sigma^{2} / S$, where $\mu_{F}^{[k, \tau]}$ is defined as

$$
\mu_{F}^{[k, \tau]}=\frac{1}{S} \sum_{l=-S N+1}^{0} P[k+l] P[k+l+\tau] .
$$

From (13), the mean value $\mu_{F}^{[k, \tau]}$ depends on $k$ and $\tau$ values. In fact, it also depends on the data pattern of the transmitted data $D[n]$. There are $(N-1)$ different values for $\tau$, which are
TABLE II

Properties of $P_{D}$ AND $P_{F}$ AS Functions of $S, T_{1}, T_{2}$, AND $\sigma$

\begin{tabular}{l|l|l}
\hline & $P_{D}$ & $P_{F}$ \\
\hline$\sigma$ & decreasing function of $\sigma$. & increasing function of $\sigma$. \\
\hline$T_{I}$ & decreasing function of $T_{1}$. & Decreasing function of $T_{1}$. \\
\hline$T_{2}$ & increasing function of $T_{2}$. & increasing function of $T_{2}$. \\
\hline$S$ & increasing function of $S$. & decreasing function of $S$. \\
\hline
\end{tabular}

$S, 2 S, \ldots, S(N-1)$, and there are four different data patterns, which are "00," "01," "10," and " 11 ." Therefore, there are total $4(N-1)$ different values for $\mu_{F}^{[k, \tau]}$. We denoted these $4(N-1)$ different mean values as $\mu_{i}$, where $i=0,1, \ldots, 4 N-5$. Let us assume that the probabilities of these $4(N-1)$ different events are the same, which is equal to $1 / 4(N-1)$. In this condition, the distribution of $Z[k]$ is $G\left(\mu, N \sigma^{2} / S\right)$ for $i=0,1, \ldots, 4 N-5$ in the AWGN channel for each event and the probability for each event $i$ is $1 / 4(N-1)$. Therefore, the probability of the false alarm $P_{F}$ can be expressed as

$$
\begin{aligned}
P_{F}= & \operatorname{Pr}\left\{Z[k]>T_{1} \mid G_{0}\right\}+\operatorname{Pr}\left\{Z[k]<T_{2} \mid G_{0}\right\} \\
= & 4(N-1) \\
& \times \sum_{i=0}^{4 N-5}\left[1-\frac{1}{2} \operatorname{erf}\left(\frac{T_{1}-\mu_{i}}{\sqrt{\frac{2 N}{S}} \sigma}\right)+\frac{1}{2} \operatorname{erf}\left(\frac{T_{2}-\mu_{i}}{\sqrt{\frac{2 N}{S}} \sigma}\right)\right] .
\end{aligned}
$$

According to the definition of error function in (11), we can see that the $\operatorname{erf}(x)$ is a monotonic increasing function of $x$. That is to say, the larger the $x$ value, the larger the $\operatorname{erf}(x)$ is. Due to this monotonic increasing property of $\operatorname{erf}(x)$, we can easily derive that $P_{D}$ is a monotonic decreasing function of $T_{1}$ and a monotonic increasing function of $T_{2}$. As the same $P_{F}$ is also a monotonic decreasing function of $T_{1}$ and a monotonic increasing function of $T_{2}$. In the general conditions, $T_{1}-N<0$ and $T_{2}+N>0$. Therefore, $P_{D}$ is a monotonic decreasing function of channel noise level $\sigma$ and a monotonic increasing function of $S$, which is the over-sampling number in each chip period. For the similar reasons, $P_{F}$ is a monotonic increasing function of channel noise level $\sigma$ and a monotonic decreasing function of $S$. These properties of the probability of false alarm and detection are summarized in Table II. According to these monotonic properties, there exists single optimized solution of design parameters to maximize $P_{D}$ or minimize $P_{F}$. The algorithm to solve this optimization problem will be shown in the next section.

The probability of detection and false alarm versus the channel noise level $T_{1}$ and $S$ are demonstrated in Figs. 4-6, respectively. The PN sequence applied to this analysis is the 11-length Barker code sequence. The distributions shown in Fig. 4 is calculated under the condition that $T_{1}=8, T_{2}=-8$, and $S=8$. The signal-to-noise ratio (SNR) value ranges from -15 to $15 \mathrm{~dB}$. It is easy to verify that $P_{D}$ is a monotonic increasing function of SNR and, therefore, is a monotonic decreasing function of channel noise level. The distributions plotted in Fig. 5 is calculated under the condition that $T_{2}=-8$ 


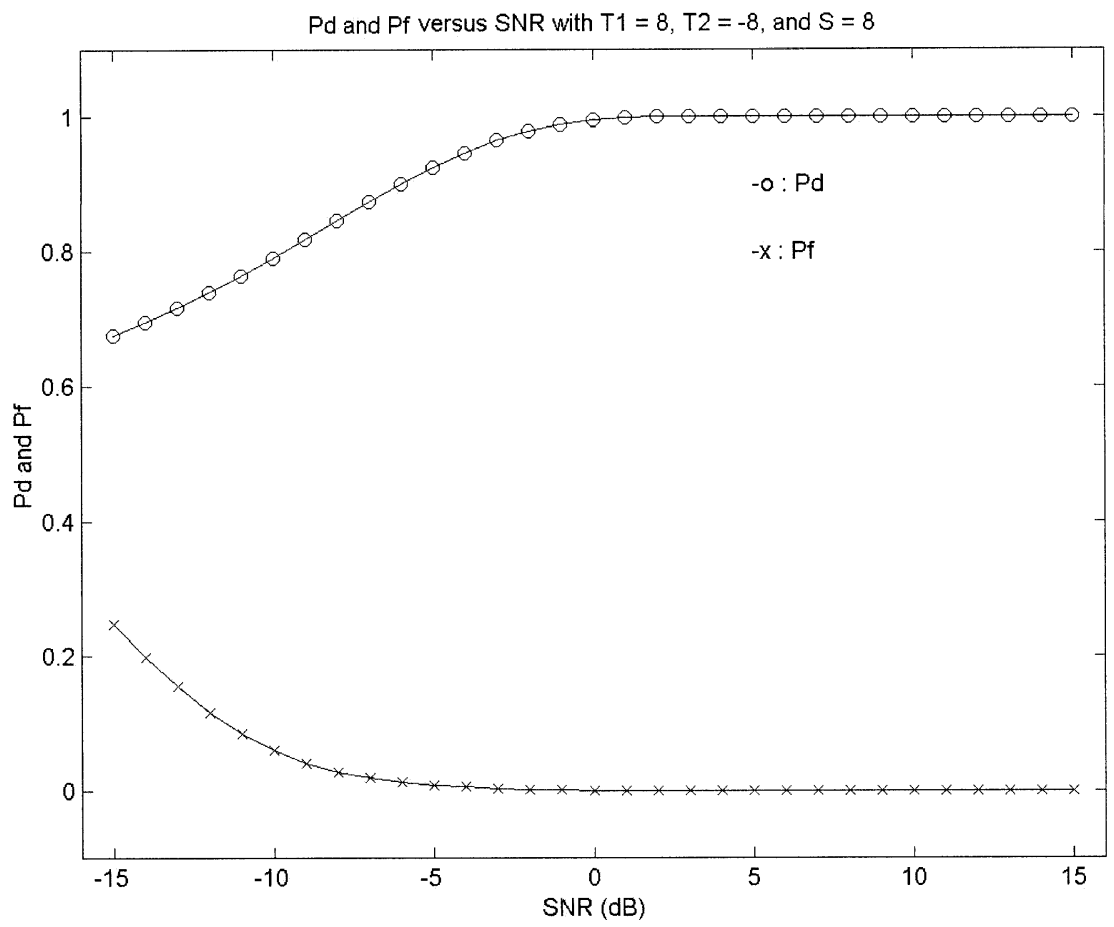

Fig. 4. Probability distributions of $P_{D}$ and $P_{F}$ as functions of SNR value.

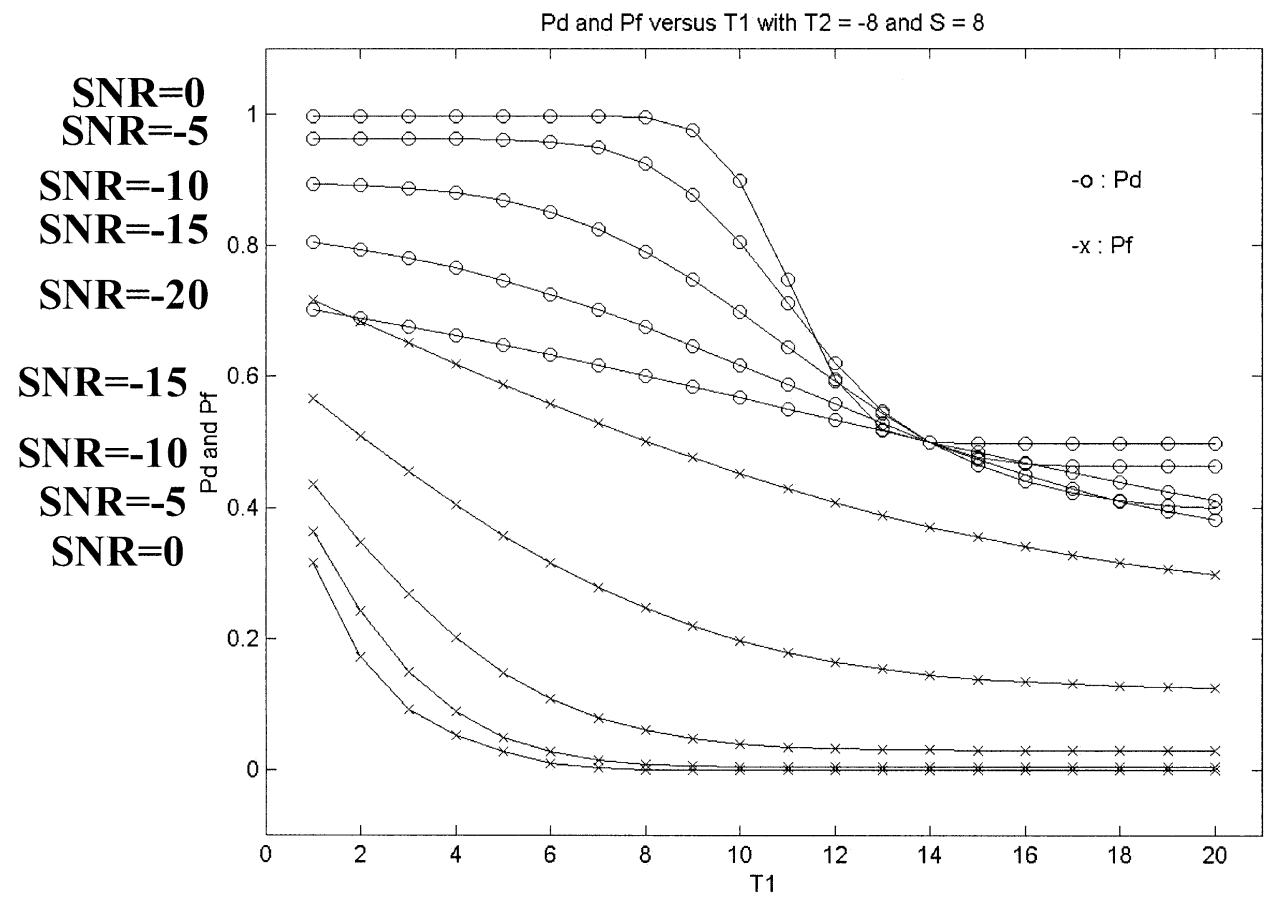

Fig. 5. Probability distributions of $P_{D}$ and $P_{F}$ as functions of $T_{1}$ with several SNR values.

and $S=8$. The $T_{1}$ value ranges from 1 to 20 . The conditions for different SNR values are also plotted in this figure. It can be verified from the figure that $P_{D}$ and $P_{F}$ are both monotonic decreasing functions of $T_{1}$. The effect of the SNR value is also disclosed in this figure. From Fig. 6, $P_{D}$ is a monotonic increasing function of over-over-sampling number $S$ under every different SNR values. The calculation of this figure is based on the condition that $T_{1}=8$ and $T_{2}=-8$. The SNR value ranges from -20 to $0 \mathrm{~dB}$ and the $S$ value ranges from 1 to $20 \mathrm{~dB}$.

\section{B. Power Consumption Analysis}

As mentioned in (1), the dynamic power consumption of digital VLSI system depends on switching frequency $f_{\mathrm{sw}}$. For the proposed PN code acquisition in Fig. 2, the switching frequency of the acquisition structure is $f_{s}=S f_{c}$, which is proportional to the sampling rate and chip frequency $f_{c}$. For given PN sequence and communication system architecture, chip frequency can be decided. Therefore, the power consumption of the acquisition structure is proportional to the over-sampling number $S$. 


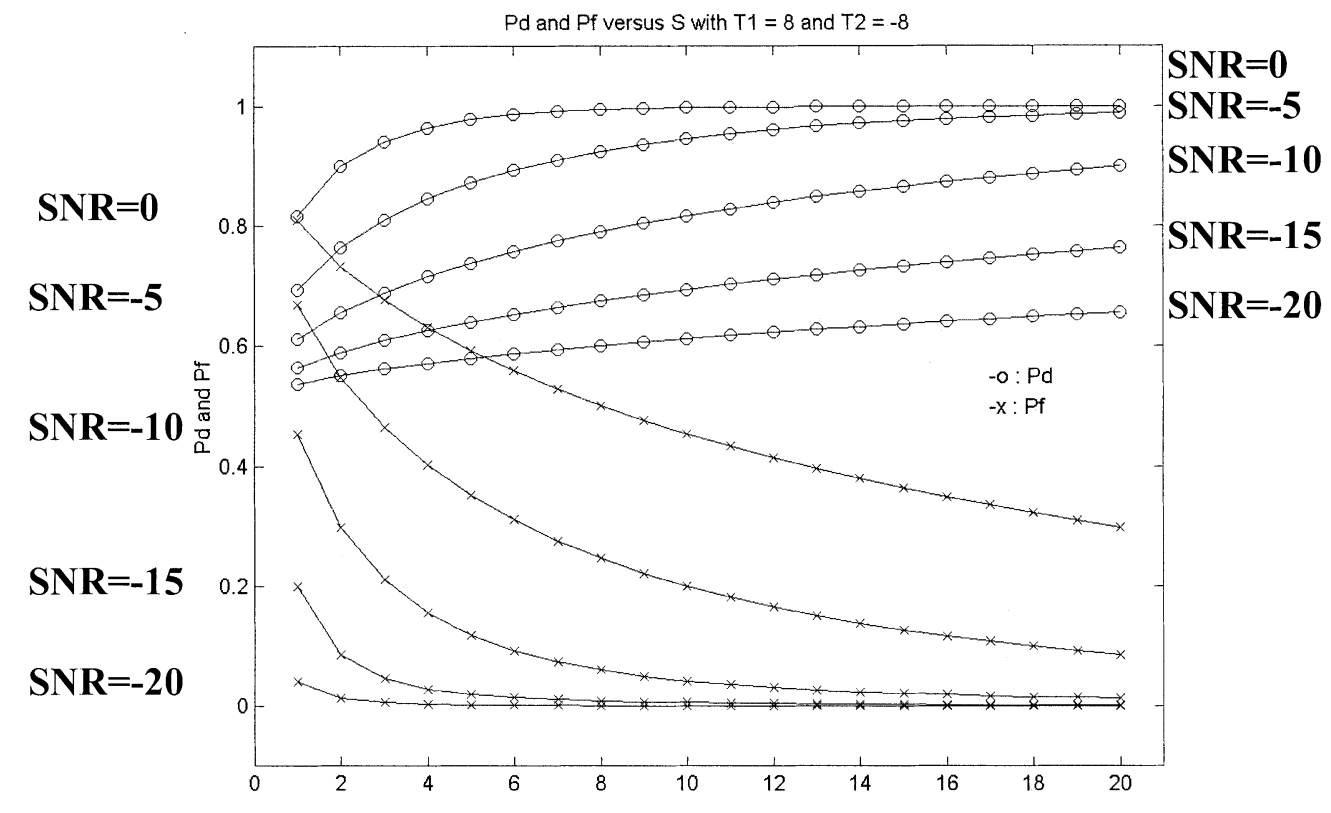

Fig. 6. Probability distributions of $P_{D}$ and $P_{F}$ as functions of $S$ with several SNR values.

For the power management module, the functional blocks of noise estimator, sampling rate generator, and threshold generator are included in this module. The operating frequencies of sampling rate generator and threshold generator are exactly the refresh rate of the noise estimator, which is denoted by $f_{R}$. $f_{R}$ is the rate at which the noise estimator update the estimated channel noise level. The refresh frequency depends on the coherent time of the channel. For example, the refresh frequency of the system with fast-fading channel will be larger than that with slow-fading channel. According to this reason, we assume that the channel is slow-fading channel. There are several practical considerations when designing the refresh rate. The data rate is usually several times of refresh rate. And the sampling rate is also several times of data rate. Because the switch frequency of the power management module is much less than that of the PN acquisition structure, the power consumption of this module can be ignored. Therefore, the power consumption of this proposed architecture is dominated by the code acquisition structure, which is mainly depends on the sampling rate.

Therefore, by controlling the sampling rate and the decision threshold dynamically according to the estimated channel noise level, the power consumption of the system can be reduced and the performance is maintained above some specifications. In the next section, we proposed a novel algorithm to control the sampling rate and threshold of the proposed PN code acquisition system to minimize the power consumption without degradation of the system performance.

\section{The Proposed Adaptive Sampling Rate and THRESHOLD CONTROL (ASTC) ALgORITHM}

In this section, the algorithm proposed to control the sampling rate and the threshold of the PN code acquisition system is described in detail. we assume that the channel noise $n[k]$ is additive white Gaussian noise with zero mean and variance $\sigma^{2}$.
We assume that the SNR value of received baseband signal is $\rho \mathrm{dB}$. The SNR value of the system can be related to $\sigma^{2}$ by

$$
\rho=10 \times \log _{10}\left(\frac{1}{\sigma^{2}}\right)
$$

For given channel noise $\sigma^{2}$ and the over-sampling number in each chip period $S$, the optimal threshold of the detector can be decided by the optimized threshold decision (OTD) algorithm proposed in [29]. The brief introduction of OTD algorithm is included in the Appendix. The OTD algorithm can calculate the threshold of a code acquisition system to make the probability of detection maximal and the probability of false alarm equal to a specific constant. The optimized threshold values $T_{1}$ and $T_{2}$, found by OTD algorithm has the relationship of $T_{2}=-T_{1}$.

In order to implement the sampling rate and threshold lookup tables, the OTD algorithm is applied to calculate the optimal threshold $T m$ and the maximal probability of detection $P_{D}$ for given SNR $\rho \mathrm{dB}$, over-sampling number $S$, and the specific probability of false alarm $P_{F}$. In the following analysis and simulation, we take 11-length Barker code as the PN sequence. Let us take an example of $\rho=0 \mathrm{~dB}, S=1$, and $P_{F}=0.01$. The thresholds and the maximal probability of detection calculated by the OTD algorithm are $T_{1}=10.1795, T_{2}=-10.1795$, and $P_{D}=0.5977$. The thresholds and the maximal probability of detection for each SNR and over-sampling number values should be calculated at first. For each SNR value in the range -10 to $10 \mathrm{~dB}$, the threshold and the probability of detection as functions of SNR and over-sampling number should be calculated by OTD algorithm. The SNR range of -10 to $10 \mathrm{~dB}$ is flexible. For larger range of SNR values, the system performance will be improved at the cost of system complexity.

In Table III, the calculated thresholds and the maximal probability of detection for $\mathrm{SNR}=-1 \mathrm{~dB}, S$ varying from 1 to 11 , and $P_{F}=0.01$ are listed for illustration. The threshold of $T_{1}$ decreases when the over-sampling number $S$ is increasing. 
TABLE III

Calculated Threshold and MaXimal Probability of DETECTION FOR SNR $=-1$ AND $P_{F}=0.01$

\begin{tabular}{r|r|r|r}
\hline $\mathbf{S}$ & \multicolumn{1}{|l|}{$\boldsymbol{T}_{1}$} & \multicolumn{1}{l}{$\boldsymbol{T}_{2}$} & \multicolumn{1}{l}{$P_{D}$} \\
\hline 1 & 11.0476 & -11.048 & 0.4949 \\
\hline 2 & 8.8125 & -8.8125 & 0.7971 \\
\hline 3 & 7.9551 & -7.9551 & 0.9218 \\
\hline 4 & 7.4927 & -7.4927 & 0.9703 \\
\hline 5 & 7.1972 & -7.1972 & 0.9888 \\
\hline 6 & 6.988 & -6.988 & 0.9959 \\
\hline 7 & 6.8299 & -6.8299 & 0.9985 \\
\hline 8 & 6.705 & -6.705 & 0.9995 \\
\hline 9 & 6.6029 & -6.6029 & 0.9998 \\
\hline 10 & 6.5176 & -6.5176 & 0.9999 \\
\hline 11 & 6.4448 & -6.4448 & 1 \\
\hline
\end{tabular}

The probability of detection increases when the over-sampling number is increasing. By the values listed in Table III, the sampling rate of the code acquisition system can be choosen to meet some specific probability of detection for given $\mathrm{SNR}=-1 \mathrm{~dB}$. For example, if the performance specifications of the acquisition system are $P_{F} \leq 0.01$ and $P_{D} \geq 0.90$, then the over-sampling number $S$ should be three to meet the specifications. Under this condition, the probability of false alarm is 0.01 and the probability of detection is 0.9218 . If the probability of detection is required to be larger than 0.99 , then the over-sampling number should be six and the probability of detection is 0.9959 . The thresholds are also adaptive due to the over-sampling number and the SNR value.

With Table III for each SNR values, the lookup tables implemented in the PN code acquisition structure can be formed. We assume that the specifications of the system performance are $P_{F} \leq 0.01$ and $P_{D} \geq 0.90$. The over-sampling number, threshold, probability of false alarm, and probability of detection are listed in Table IV as functions of SNR value. For each SNR value, the values in Table IV can be calculated from the tables such as Table III. The over-sampling number decreases when the SNR value is increasing. At the same time, the probability of detection is always larger than 0.90 , which meets the system specifications.

The proposed ASTC algorithm is described in the following.

Step 1: Given the system specifications of the performance of the proposed PN code acquisition system.

$$
\left\{\begin{array}{l}
P_{D}\left(\mathrm{SNR}, S, T_{1}, T_{2}\right) \geq P_{D, \text { spec }} \\
P_{F}\left(\mathrm{SNR}, S, T_{1}, T_{2}\right) \leq P_{F, \text { spec }}
\end{array}\right.
$$

where $P_{D \text {,spec }}$ is the minimum specification of the probability of detection and $P_{F, \text { spec }}$ is the maximal specification of the probability of false alarm.

Step 2: Set the ranges and resolutions of the SNR value as

$$
R_{\mathrm{SNR}}=\left\{x \in Z \mid \mathrm{SNR}_{\min } \leq x \leq \mathrm{SNR}_{\max }\right\}
$$

Step 3: For each $\rho \mathrm{dB} \in R_{\mathrm{SNR}}$, calculate the minimal over-sampling number $S(\rho)$ and
TABLE IV

LOOKUP TABLES IMPLEMENTED IN THE CODE ACQUiSITION SySTEM With the Specifications of $P_{F} \leq 0.01$ and $P_{D} \geq 0.90$

\begin{tabular}{r|r|r|r|r|l}
\hline $\begin{array}{l}\text { SNR(d } \\
\text { B) }\end{array}$ & $\boldsymbol{S}$ & \multicolumn{1}{l}{$\boldsymbol{T}_{\boldsymbol{l}}$} & \multicolumn{1}{l}{$\boldsymbol{T}_{2}$} & \multicolumn{1}{l}{$P_{D}$} & $P_{F}$ \\
\hline-10 & 22 & 8.103 & -8.103 & 0.9024 & 0.010 \\
\hline-9 & 18 & 8.0472 & -8.0472 & 0.9099 & 0.010 \\
\hline-8 & 14 & 8.0868 & -8.0868 & 0.9046 & 0.010 \\
\hline-7 & 11 & 8.1076 & -8.1076 & 0.9018 & 0.010 \\
\hline-6 & 9 & 8.0516 & -8.0516 & 0.9093 & 0.010 \\
\hline-5 & 7 & 8.0913 & -8.0913 & 0.904 & 0.010 \\
\hline-4 & 6 & 7.9508 & -7.9508 & 0.9223 & 0.010 \\
\hline-3 & 5 & 7.8665 & -7.8665 & 0.9326 & 0.010 \\
\hline-2 & 4 & 7.8543 & -7.8543 & 0.9341 & 0.010 \\
\hline-1 & 3 & 7.9551 & -7.9551 & 0.9218 & 0.010 \\
\hline 0 & 3 & 7.577 & -7.577 & 0.9631 & 0.010 \\
\hline 1 & 2 & 7.8583 & -7.8583 & 0.9336 & 0.010 \\
\hline 2 & 2 & 7.4961 & -7.4961 & 0.97 & 0.010 \\
\hline 3 & 2 & 7.1914 & -7.1914 & 0.9891 & 0.010 \\
\hline 4 & 1 & 7.8624 & -7.8624 & 0.9331 & 0.010 \\
\hline 5 & 1 & 7.4995 & -7.4995 & 0.9697 & 0.010 \\
\hline 6 & 1 & 7.1943 & -7.1943 & 0.989 & 0.010 \\
\hline 7 & 1 & 6.9346 & -6.9346 & 0.997 & 0.010 \\
\hline 8 & 1 & 6.7113 & -6.7113 & 0.9994 & 0.010 \\
\hline 9 & 1 & 6.5176 & -6.5176 & 0.9999 & 0.010 \\
\hline 10 & 1 & 6.3483 & -6.3483 & 1 & 0.010 \\
\hline & & & &
\end{tabular}

the corresponding thresholds $T_{1}(\rho) T_{2}(\rho)$ by the OTD algorithm, such that the following conditions hold:

$$
\left\{\begin{array}{l}
P_{D}\left(\rho \mathrm{dB}, S(\rho \mathrm{dB}), T_{1}(\rho \mathrm{dB}), T_{2}(\rho \mathrm{dB})\right) \geq P_{D, \text { spec }} \\
P_{F}\left(\rho \mathrm{dB}, S(\rho \mathrm{dB}), T_{1}(\rho \mathrm{dB}), T_{2}(\rho \mathrm{dB})\right)=P_{F, \text { spec }}
\end{array}\right.
$$

Step 4: Create the lookup tables of over-sampling number and threshold of Step 3 in the proposed PN code acquisition system.

In the following, we take an example to demonstrate the operation of the lookup table implemented in the blocks of "sampling rate generator" and "threshold generator", which is shown in Fig. 2. We assume that the specifications of the system performance are $P_{F} \leq 0.01$ and $P_{D} \geq 0.90$. The lookup table implemented in the proposed system is listed in Table IV. We denote the over-sampling number $S$ and the threshold $T_{1}$ as $S(\mathrm{SNR})$ and $T_{1}(\mathrm{SNR})$, which emphasize that they are functions of SNR value. For the optimized solution $T_{1}$ and $T_{2}$ found by OTD, they meet the relationship $T_{2}=-T_{1}$, when the channel noise is AWGN. Let $\mathrm{SNR}_{\mathrm{EST}}$ be the estimated SNR value, which is the output of the noise estimator in Fig. 2. This SNR $_{\text {EST }}$ will be sent to the blocks of "sampling rate generator" and "threshold generator" to generate the over-sampling number $S$ and the threshold $T_{1}$. These two values will be generated by (19) shown at the bottom of the next page, where $S(n \mathrm{~dB})$ is the $S$ value listed in the row at $\mathrm{SNR}=n \mathrm{~dB}$ in Table V. For example, $S(-6 \mathrm{~dB})=9$ shown in (20) at the bottom of the next page, where $T_{1}(n \mathrm{~dB})$ and $T_{2}(n \mathrm{~dB})$ is the $T_{1}$ and $T_{2}$ value listed in the row at $\mathrm{SNR}=n \mathrm{~dB}$ in Table V, respectively. For example, $T_{1}(-6 \mathrm{~dB})=8.0516$. In 
TABLE $\mathrm{V}$

The Simulation Results OF THE PaRAmeters of the PROPOSED SYSTEM AND THE CONVENTIONAL SYSTEM WITH THE SPECIFICATIONS OF $P_{F} \leq 0.01$ AND $P_{D} \geq 0.90$

\begin{tabular}{l|r|r}
\hline & Proposed & Conventional \\
\hline$S$ & 2.655 & 9 \\
\hline $\boldsymbol{P}_{F, \max }$ & 0.01 & 0.0222 \\
\hline $\boldsymbol{P}_{D, \min }$ & 0.902 & 0.8616 \\
\hline
\end{tabular}

the following, we will prove that the system performance will be guaranteed to meet the system specifications due to the (19) and (20) except the condition when SNR $\mathrm{EST}_{\text {is less than }}$ $-10 \mathrm{~dB}$. However, the probability of the event that $\mathrm{SNR}_{\mathrm{EST}}$ is less than $-10 \mathrm{~dB}$ is very low. In the following discussion, we denote the probability of detection and the probability of false alarm by (21) and (22), respectively

$$
\begin{aligned}
& P_{D}=P_{D}\left(\mathrm{SNR}, S, T_{1}, T_{2}\right) \\
& P_{F}=P_{F}\left(\mathrm{SNR}, S, T_{1}, T_{2}\right) .
\end{aligned}
$$

Assume that the estimated value $\mathrm{SNR}_{\mathrm{EST}}$ is in the range of

$$
n \mathrm{~dB} \leq \mathrm{SNR}_{\mathrm{EST}}<(n+1) \mathrm{dB}, \quad n=-10, \ldots, 9 .
$$

Then the probability of detection of the proposed system becomes

$$
\begin{aligned}
P_{D}= & P_{D}\left(\mathrm{SNR}_{\mathrm{EST}}, S\left(\mathrm{SNR}_{\mathrm{EST}}\right), T_{1}\left(\mathrm{SNR}_{\mathrm{EST}}\right)\right. \\
& \left.T_{2}\left(\mathrm{SNR}_{\mathrm{EST}}\right)\right) \\
= & P_{D}\left(\mathrm{SNR}_{\mathrm{EST}}, S(n \mathrm{~dB}), T_{1}(n \mathrm{~dB}), T_{2}(n \mathrm{~dB})\right)
\end{aligned}
$$

where $S(n \mathrm{~dB}), T_{1}(n \mathrm{~dB})$ and $T_{2}(n \mathrm{~dB})$ are the values in Table IV. By the characteristics of Table IV, the following equation is always true:

$$
P_{D}\left(n \mathrm{~dB}, S(n \mathrm{~dB}), T_{1}(n \mathrm{~dB}), T_{2}(n \mathrm{~dB})\right) \geq P_{D, \text { spec }}=0.90
$$

where $P_{D \text {,spec }}$ is the minimum specification of the probability of detection. From Table II, the probability of detection is the decreasing function of $\sigma$ and $\sigma$ is the decreasing function of SNR value by (15). Therefore, the probability of detection is the increasing function of SNR value. By applying this characteristics of the probability of detection, the probability of detection can be proved to meet the system specifications in the proposed system by

$$
\begin{aligned}
P_{D} & =P_{D}\left(\mathrm{SNR}_{\mathrm{EST}}, S(n \mathrm{~dB}), T_{1}(n \mathrm{~dB}), T_{2}(n \mathrm{~dB})\right) \\
& \geq P_{D}\left(n \mathrm{~dB}, S(n \mathrm{~dB}), T_{1}(n \mathrm{~dB}), T_{2}(n \mathrm{~dB})\right) \geq P_{D, \text { spec }} \\
& =0.90 \text { because } \mathrm{SNR}_{\mathrm{EST}} \geq n \mathrm{~dB} .
\end{aligned}
$$

For the condition of $10 \mathrm{~dB} \leq \mathrm{SNR}_{\mathrm{EST}}$, it is trivial that the proposed system will meet the system specifications under this condition. By the similar derivations, the probability of false alarm of the proposed system can be proved to always meet the system performance specifications. Therefore, by applying the lookup table in Table IV into the PN code acquisition system, a low-power system with controllable system performance can be implemented.

\section{The Simulations of POWER CONSUmption AND SYSTEM PERFORMANCE}

The simulation results for the proposed PN code acquisition system and the conventional code acquisition system are presented in this section.

We assume that the specifications of the system performance are $P_{F} \leq 0.01$ and $P_{D} \geq 0.90$. The lookup table of Table IV is used in the proposed structure. In the simulations described below, we assume that the channel is a time-varying AWGN channel. The channel noise have the Gaussian distribution $G\left(0, \sigma^{2}\right)$, the Gaussian distribution with mean zero and variance $\sigma^{2}$. However, the variance $\sigma^{2}$ is not a constant for the real channel condition and it is time-varying. Therefore, we model $\sigma^{2}$ as a random variable and the distribution of $\sigma^{2}$ is modeled as the random variable $\Sigma_{N} . \Sigma_{N}$ is modeled as the square of one Gussian distribution, which is expressed as

$$
\Sigma_{N}=N^{2}
$$

where $N$ is the Gaussian distribution with $G(0,1)$. We assume that the channel is a slow fading channel. With the appropriate design of the refresh frequency $f_{R}$ of the noise estimator, the variance of the channel noise $\sigma^{2}$ will nearly keep the

$$
S\left(\mathrm{SNR}_{\mathrm{EST}}\right)= \begin{cases}S(-10 \mathrm{~dB}) & \mathrm{SNR}_{\mathrm{EST}}<-10 \mathrm{~dB} \\ S(n \mathrm{~dB}) & n \mathrm{~dB} \leq \mathrm{SNR}_{\mathrm{EST}}<(n+1) \mathrm{dB}, \quad n=-10, \ldots, 9 \\ S(10 \mathrm{~dB}) & 10 \mathrm{~dB} \leq \mathrm{SNR}_{\mathrm{EST}}\end{cases}
$$

$$
\begin{aligned}
T_{1}\left(\mathrm{SNR}_{\mathrm{EST}}\right) & =\left\{\begin{array}{lll}
T_{1}(-10 \mathrm{~dB}) & \mathrm{SNR}_{\mathrm{EST}}<-10 \mathrm{~dB} \\
T_{1}(n \mathrm{~dB}) & n \mathrm{~dB} \leq \mathrm{SNR}_{\mathrm{EST}}<(n+1) \mathrm{dB}, & n=-10, \ldots, 9 \\
T_{1}(10 \mathrm{~dB}) & 10 \mathrm{~dB} \leq \mathrm{SNR}_{\mathrm{EST}}
\end{array}\right. \\
T_{2}\left(\mathrm{SNR}_{\mathrm{EST}}\right) & =\left\{\begin{array}{lll}
T_{2}(-10 \mathrm{~dB}) & \mathrm{SNR}_{\mathrm{EST}}<-10 \mathrm{~dB} \\
T_{2}(n \mathrm{~dB}) & n \mathrm{~dB} \leq \mathrm{SNR}_{\mathrm{EST}}<(n+1) \mathrm{dB}, & n=-10, \ldots, 9 \\
T_{2}(10 \mathrm{~dB}) & 10 \mathrm{~dB} \leq \mathrm{SNR}_{\mathrm{EST}}
\end{array}\right.
\end{aligned}
$$




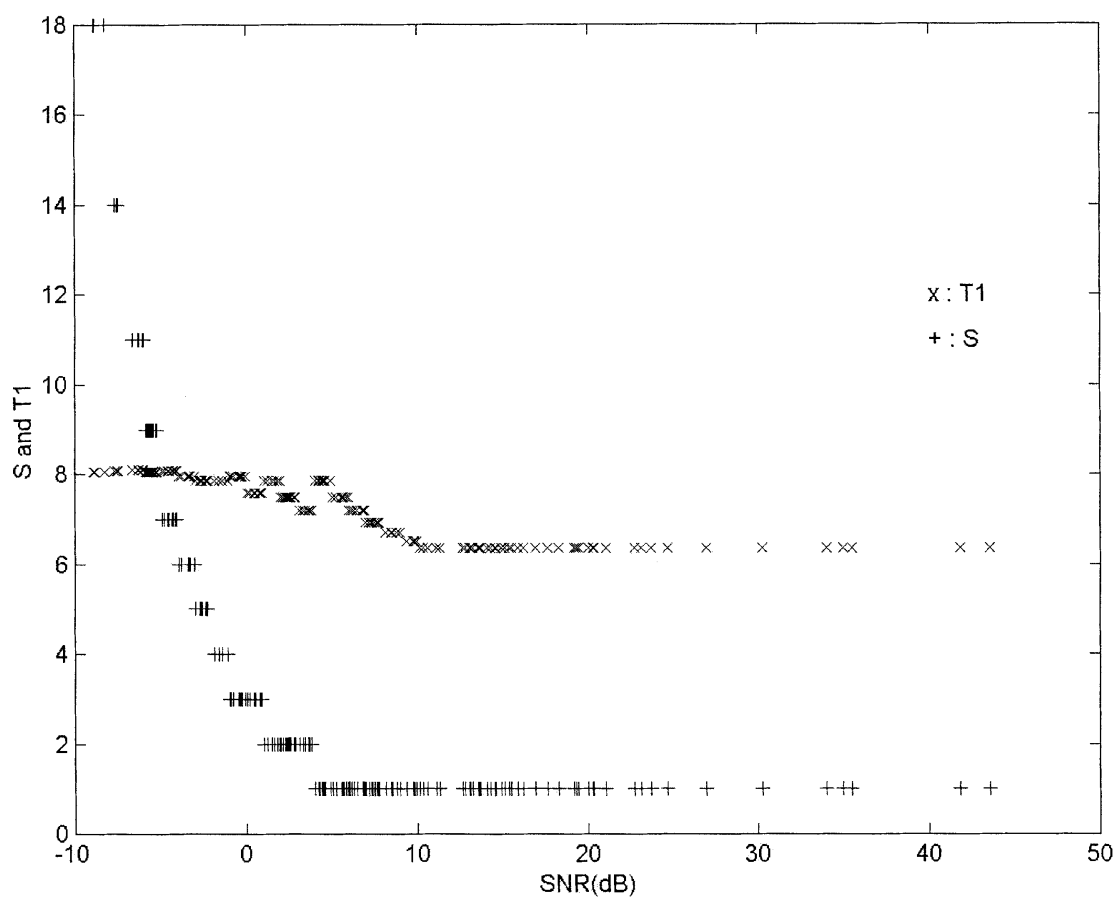

Fig. 7. Distributions of oversampling number $S$ and threshold $T_{1}$ versus the SNR value.

same within one refresh period, which is $T_{R}=1 / f_{R}$. The channel can be viewed as a time-invariant AWGN channel with $G\left(0, \sigma_{i}^{2}\right)$ within one refresh period, where $\sigma_{i}^{2}$ is the variance of the channel noise within the $i$ th refresh period. The distributions of $P_{D}$ and $P_{F}$ derived in (10) and (14) can be applied to the analysis of the system performance within one refresh period. The variance of the channel noise may be different in different refresh periods, with the distribution modeled in (27). For the proposed architecture, the noise estimator will estimate the noise variance $\sigma_{i}^{2}$ and update the sampling rate and the threshold of the proposed system according to the estimated variance.

The values of the over-sampling number $S$ and the threshold $T_{1}$ versus SNR are plotted in Fig. 7. The SNR values in this simulation range from -10 to $44 \mathrm{~dB}$ and 200 different SNR values are included in this simulation. When the SNR value is low, which means the channel noise level is high, the over-sampling number $S$, which represents the sampling rate $f_{S}$, is adaptively tuned to a high level to compensate the high channel noise level. At the same time, the threshold is updated to optimize the system performance. The dynamic range of the threshold value is not as wide as that of the over-sampling number. The updates of over-sampling number and the threshold follow the equations of (19) and (20) by the functional blocks of sampling rate generator and threshold generator in Fig. 2. The probability of detection and the probability of false alarm for this simulation are shown in Figs. 8 and 9, respectively. From these two figures, these two probabilities have been verified to meet the system specifications. The probability of detection is always larger than 0.90 . The probability of false alarm is always less than 0.01 .

For simulations of the power consumption, several parameters are defined to analyze the proposed system and compare it to the conventional code acquisition system with fixed sampling rate and threshold. $\bar{S}$ is the average value of the over-sampling numbers. $P_{F, \max }$ and $P_{D, \min }$ are the maximal value of probability of false alarm and the minimal value of probability of detection, respectively. In the analysis in Section III-B, the power consumption of the proposed system is proportional to the sampling rate, which is the linear function of the over-sampling number. Therefore, one system with lower average over-sampling number has the lower power consumption.

For the simulation of the conventional code acquisition system, the conventional code acquisition system is designed to meet the system specifications at the $95 \%$ of the period. By (27), the variance of the channel noise is the square of $N$, which is Gaussian distribution with mean zero and variance one. Due to $N$ is Gaussian distribution, $\operatorname{Pr}\{-1.96<N<1.96\}=0.95$. For $95 \%$ of the period, the variance of the channel noise will be less than $(1.96)^{2}=3.8416=-5.85 \mathrm{~dB}$. By observing Table IV, the over-sampling number of the conventional system is set at nine and the thresholds are set to -8 and 8 . Equation (10) and (14) are calculated to verify that the system meets the $P_{D}$ and $P_{F}$ specifications at SNR $=-5.85 \mathrm{~dB}$. Therefore, the conventional system will meet the performance specifications for $95 \%$ of the period.

In Table $\mathrm{V}$, the values of $\bar{S}, P_{F, \max }$, and $P_{D \text {, min }}$ of the proposed and the conventional systems are listed for comparison. For the proposed one, the average over-sampling number is 2.655 , which is only $29.5 \%$ of the over-sampling number of the conventional one. The over-sampling number of the conventional one is nine. Therefore, the power consumption of the proposed one is only about $30 \%$ of the conventional one. About $70 \%$ of the power consumption is reduced by applying the proposed code acquisition structure. The maximal probability of false alarm for the proposed one is 0.01 , which is no larger than the specifications. However, this is not the 


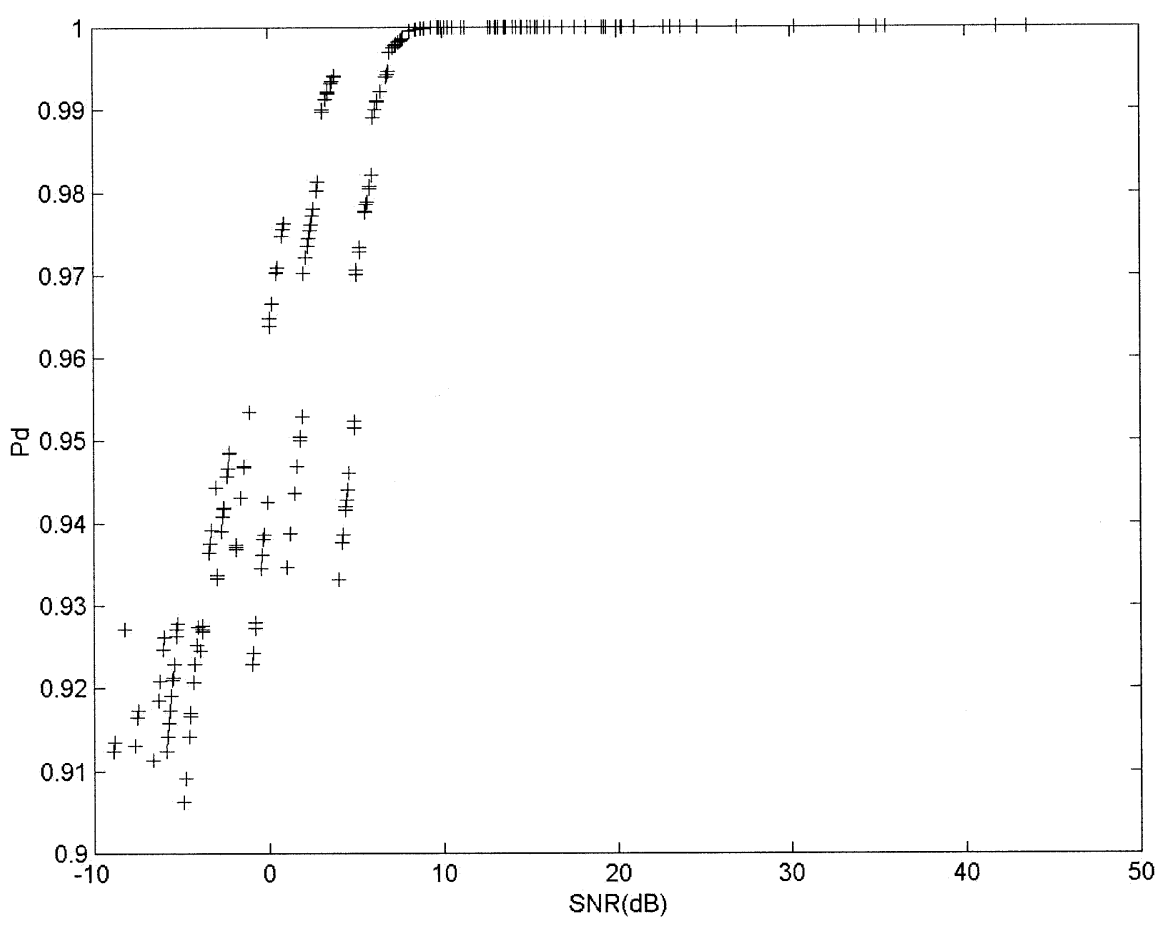

Fig. 8. Probability of detection $P_{D}$ versus the SNR value.

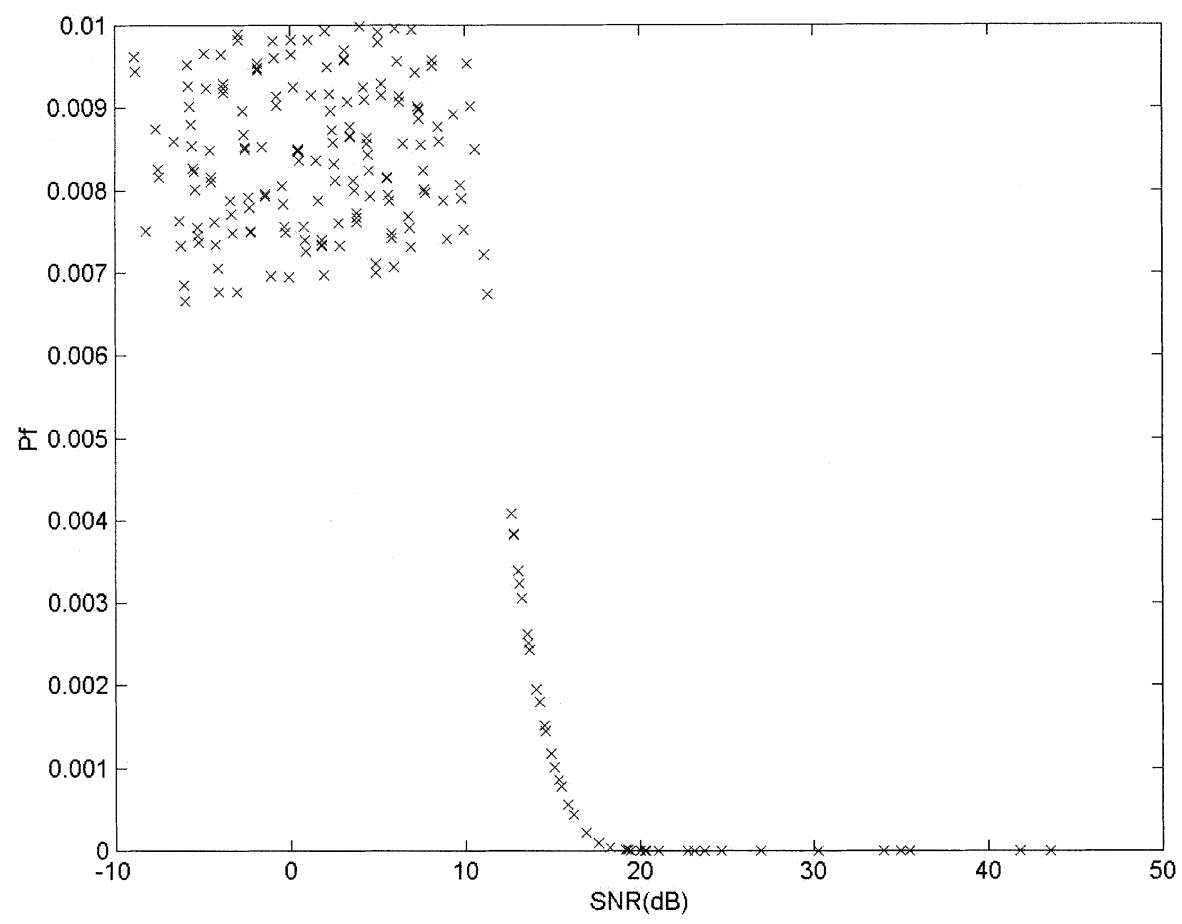

Fig. 9. Probability of false alarm $P_{F}$ versus the SNR value.

case for the conventional one. The maximal probability of false alarm for the conventional one is 0.222 , which is larger than the specifications. The reason is that the conventional system only meets the specifications for $95 \%$ of the period. There are similar conditions for the probability of detection.

More simulation cases were done to show the low-power properties of the proposed system. In Table VI, ten cases of the simulation conditions are summarized. These simulation cases are with different maximal specification of the probability of false alarm $P_{F \text {,spec }}$ and different minimal specification of the probability of detection $P_{D \text {,spec }}$. The values of $P_{F \text {,spec }}$ are 0.005 and 0.01 and the values of $P_{D \text {,spec }}$ are $0.8,0.85,0.9$, 0.95 , and 0.99 . The average over-sampling numbers $\bar{S}$ of the proposed system are also shown in the table for each simulation case. The fixed over-sampling numbers $S_{\text {conv }}$ of the conventional system are also listed in the table for each simulation case. The percentages of the reduction of power consumption $P_{P, r e}$ for the proposed system compared to the corresponding 
TABLE VI

THE SimUlation RESUlts OF THE POWER CONSUMPTION COMPARISON OF THE PROPOSED SYSTEM AND THE CONVENTIONAL SYSTEM FOR THE TEN Simulatoin CASES

\begin{tabular}{l|r|r|r|r|l}
\hline & $\boldsymbol{P}_{\boldsymbol{F} \text {,spec }}$ & $\boldsymbol{P}_{d \text { dspec }}$ & $\bar{S}$ & $\boldsymbol{S}_{\text {conv }}$ & $\mathbf{P}_{P, \text { re }}$ \\
\hline Case 1 & 0.005 & 0.8 & 2.645 & 8 & $66.9 \%$ \\
\hline Case 2 & 0.005 & 0.85 & 2.925 & 10 & $70.8 \%$ \\
\hline Case 3 & 0.005 & 0.9 & 3.350 & 11 & $69.5 \%$ \\
\hline Case 4 & 0.005 & 0.95 & 3.600 & 14 & $74.3 \%$ \\
\hline Case 5 & 0.005 & 0.99 & 5.255 & 20 & $73.7 \%$ \\
\hline Case 6 & 0.01 & 0.8 & 2.305 & 7 & $67.1 \%$ \\
\hline Case 7 & 0.01 & 0.85 & 2.380 & 8 & $70.3 \%$ \\
\hline Case 8 & 0.01 & 0.9 & 2.655 & 9 & $70.5 \%$ \\
\hline Case 9 & 0.01 & 0.95 & 3.355 & 11 & $69.5 \%$ \\
\hline Case 10 & 0.01 & 0.99 & 4.755 & 17 & $72.0 \%$ \\
\hline
\end{tabular}

conventional one are shown in the last row of the table. For all the ten cases, the proposed systems reduce about $70 \%$ of the power consumption when compared to the conventional one. For the fourth case, the percentage of the power consumption reduction is even $74.3 \%$. All the ten simulation cases show that the proposed system is an power-efficient system and it can be applied to a large range of system performance specifications.

\section{EXPANSION AND LIMITATION}

The analysis and simulation of the proposed ASTC algorithm are based on the assumption that the channel noise is known and distributed as the AWGN channel. However, under the assumptions that the probability distribution of channel noise is known and the mean and variance of the channel noise could be estimated, the proposed ASTC algorithm can be easily expanded to spread-spectrum system under arbitrary channel noise distribution functions. This is demonstrated in the Appendix.

The power consumption analysis performed in this paper is focused on the PN code acquisition system, which is just one part of the entire wireless communication system. The power consumption of the entire wireless system depends on the architecture of the entire wireless system and what applications the system applied. System architecture issues such as hardware software partition, processor or DSP module implementation, and RF front-end architecture will have effects to how much power the PN acquisition system consumes with respect to the entire wireless system. For general case, the wireless receiver is one of the most complex parts in the wireless hardware design. Clock recovery and synchronization circuits are two of the most complex parts in the receiver design. Therefore, the PN acquisition system dissipates large percentage of power consumption with respect to the entire wireless system. However, it is difficult to declare a exact percentage.

\section{CONCLUSION}

By adaptively controlling the over-sampling number and threshold of the proposed PN code acquisition system, the power consumption is greatly reduced when compared to a conventional structure with fixed sampling rate and threshold. At the same time, the system performance of the proposed system also meets the system specifications. Simulations of a large range of the system specifications have revealed that the proposed system consumes $70 \%$ less power than the conventional one. It had been shown that the proposed adaptive code acquisition architecture combined with the proposed algorithm is an efficient design methodology for the low-power spread-spectrum communication systems.

\section{APPENDIX}

The brief introduction of the OTD algorithm and the expansion of the proposed architecture to the non-AWGN channel are included in this appendix.

\section{A. Brief Introduction to OTD Algorithm}

The optimized system performance of a PN acquisition system can be achieved by maximizing $P_{D}$ or minimizing $P_{F}$. The OTD algorithm is one searching algorithm used to find the optimized solution of the decision threshold values to optimize the system performance. Two approaches exist for the optimal system performance. One is to maximize $P_{D}$ and keep $P_{F}$ as constant. Another is to minimize $P_{F}$ and keep $P_{D}$ as constant. When given the SNR value and over-sampling number $S$, we can express $P_{D}$ and $P_{F}$ as functions of $T_{1}$ and $T_{2}$, which are shown in (A.1) and (A.2), respectively

$$
\begin{aligned}
& P_{D}=f\left(T_{1}, T_{2}\right) \\
& P_{F}=g\left(T_{1}, T_{2}\right) .
\end{aligned}
$$

The OTD algorithm is proposed to solve the following optimal problem:

$$
\begin{aligned}
& \text { Find }\left(T_{1}, T_{2}\right) \text {, such that } P_{D}=f\left(T_{1}, T_{2}\right)=f(\vec{x}) \text { is } \max \\
& \text { and } P_{F}=g\left(T_{1}, T_{2}\right)=g(\vec{x})=k_{F} \\
& \text { where } \vec{x}=\left(T_{1}, T_{2}\right) .
\end{aligned}
$$

This is an optimal problem under constrained conditions. The OTD algorithm is an iterative approach with two loops to solve this problem. The first loop is moving the initial guess of point of the second loop toward the optimized solution by moving at the direction of gradient of $f(\vec{x})$. The second loop is moving toward the constant contour line of $g(\vec{x})=k_{F}$ by Newton's method. After both two loops are converged, the optimized solution is found. The monotonic properties of $f(\vec{x})$ and $g(\vec{x})$ will guaranteed the convergence of this searching algorithm.

\section{B. The Expansion of the Proposed Architecture to Non-AWGN Channel}

Let us assume the channel noise level $n[k]$ in (3) has the following probability density function and probability accumulative function:

$$
\begin{aligned}
\operatorname{Pr}(n[k]=x) & =f_{n}(x) \geq 0, \text { where }-\infty<x<\infty \\
F_{n}(x) & =\operatorname{Pr}(n[k] \leq x) \\
& =\int_{-\infty}^{x} f_{n}(t) d t, \text { where }-\infty<x<\infty
\end{aligned}
$$


We assume that $f_{n}(x)$ is known and the mean and variance of $n[k]$ can be estimated by some methods. The mean and variance of $n[k]$ can be expressed by the following equations according to the definitions of mean and variance of a random variable:

$$
\begin{aligned}
& \mu_{n}=E(n[k])=\int_{-\infty}^{\infty} x f_{n}(x) d x \\
& \sigma_{n}^{2}=\operatorname{Var}(n[k])=\int_{-\infty}^{\infty} x^{2} f_{n}(x) d x-\mu_{n}^{2} .
\end{aligned}
$$

Let us consider the condition when $H_{1}$ and $G_{1}$ are true. The expression of $Z[k]$ in (8) can be reduced to expression in (9) under this condition. Because $n[k+l]$ in (9) are the identical independent distributions with mean $\mu_{n}$ and variance $\sigma_{n}^{2}$ and $P[k+l]= \pm 1$, which are constant values, the mean and variance of $Z[k]$ can be expressed, respectively, as follows:

$$
\begin{aligned}
\mu_{Z} & =E(Z[k])=N+\frac{1}{S} \sum_{l=-S N+1}^{0} P[k+l] \mu_{n} \\
& =N+\frac{1}{S} \times S \times t \times \mu_{n}=N+t \mu_{n} \\
\sigma_{Z}^{2} & =\operatorname{Var}(Z[k])=\frac{1}{S^{2}} \sum_{l=-S N+1}^{0} P[k+l]^{2} \sigma_{n}^{2}=\frac{N \sigma_{n}^{2}}{S}
\end{aligned}
$$

where the number one in PN sequence $P[k]$ is larger than the number zero by $t$. The value of $t$ may be positive or negative. The value of $t$ is usually near to zero for general PN sequence. Assume that $Z_{u}$ is the normalized distribution of $Z[k]$, which is correlated to $Z[k]$ by

$$
Z_{u}=\frac{Z[k]-\mu_{Z}}{\sigma_{Z}}
$$

It is easy to verify that the mean and variance of $Z_{u}$ is zero and one, respectively. We assume that the probability density function and probability accumulation function of $Z_{u}$ are $f_{Z u}(x)$ and $F_{Z u}(x)$. One part of $P_{D}$ can be expressed as

$$
\begin{aligned}
\operatorname{Pr}\left[Z[k]>T_{1} \mid H_{1} \cap G_{1}\right] & =1-F_{Z u}\left(\frac{T_{1}-\mu_{Z}}{\sigma_{Z}}\right) \\
& =1-F_{Z u}\left(\frac{T_{1}-N-t \mu_{n}}{\sqrt{\frac{N}{S \sigma_{n}}}}\right) .
\end{aligned}
$$

After similar manipulations, the probability of detection can be expressed as

$$
\begin{array}{r}
P_{D}=\frac{1}{2}\left[1-F_{Z u}\left(\frac{T_{1}-N-t \mu_{n}}{\sqrt{\frac{N}{S \sigma_{n}}}}\right)\right. \\
\left.+F_{Z u}\left(\frac{T_{2}+N+t \mu_{n}}{\sqrt{\frac{N}{S \sigma_{n}}}}\right)\right] .
\end{array}
$$

After the manipulations above, the probability of detection had been expressed as functions of over-sampling number $S$, se- quence length of PN sequence $N$, threshold values $T_{i}$, mean of channel noise $\mu_{n}$, and variance of channel noise $\sigma_{n}$. Because $F_{Z u}(x)$ is a probability accumulation function, $F_{Z u}(x)$ is a monotonic increasing function of $x$. Therefore, it is easy to verify that $P_{D}$ is a monotonic decreasing function of $T_{1}$ and a monotonic decreasing function of $T_{2}$. Under the assumptions that $T_{1}-N-t \mu_{n}<0$ and $T_{2}+N+t \mu_{n}>0, P_{D}$ is a monotonic increasing function of $S$ and a monotonic decreasing function of $\sigma_{n}$.

After similar procedures, the monotonic properties of $P_{F}$ also can be proved. Therefore, the $P_{D}$ and $P_{F}$ in non-AWGN channel also have the properties shown in Table II. Therefore, the OTD algorithm can be applied to find the optimized threshold values for given estimated mean and variance of the channel noise. The proposed architecture together with the proposed ASTC algorithm can be applied to the non-AWGN channel.

\section{REFERENCES}

[1] N. H. E. Weste and K. Eshraghian, Principles of CMOS VLSI DesignA System Perspective, 2nd ed. Reading, MA: Addison-Wesley, 1994.

[2] M. Alidina, J. Monteiro, S. Devadas, A. Ghosh, and M. Papaefthymiou, "Precomputation-based sequential logic optimization for low power," IEEE Trans. Very Large Scale Integrated Syst., vol. 2, pp. 426-436, Dec. 1994.

[3] J. S. Lee, H. D. Lee, C. W. Park, and S. Y. Hwang, "Power-conscious sheduling algorithm for performance-driven datapath synthesis," Electron. Lett., vol. 32, pp. 1574-1576, Aug. 1996.

[4] M. Winzker, "Low-power arithmetic for the processing of video signals," IEEE Trans. Very Large Scale Integrated Syst., vol. 6, pp. 493-497, Sept. 1998.

[5] C. Y. Wang and K. Roy, "An activity-driven encoding scheme for power optimization in microprogrammed control unit," IEEE Trans. Very Large Scale Integrated Syst., vol. 7, pp. 130-134, Mar. 1999.

[6] T. Xanthopoulos and A. P. Chandrakasan, "A low-power IDCT macrocell for MPEG-2 MP@ML exploiting data distribution properties for minimal activity," IEEE J. Solid-State Circuits, vol. 34, pp. 693-703, May 1999.

[7] S. Ramprasad, N. R. Shanbhag, and I. N. Hajj, "A coding framework for low-power address and data bused," IEEE Trans. Very Large Scale Integrated Syst., vol. 7, pp. 212-221, June 1999.

[8] Y. K. Seng and S. S. Rofail, "1.1 V high speed, low-power BiCMOS logic circuit," Electron. Lett., vol. 31, pp. 1039-1041, June 1995.

[9] -, "1.1 V full-swing double bootstrapped BiCMOS logic gates," IEE Proc. Circuits, Devices, Syst., vol. 143, pp. 41-45, Feb. 1996.

[10] C. C. Yeh, J. H. Lou, and J. B. Kuo, "1.5 V CMOS full-swing energy efficient logic (EEL) circuit suitable for low-voltage and low-power VLSI applications," Electron. Lett., vol. 33, pp. 1375-1376, July 1997.

[11] Y. K. Seng and S. S. Rofail, "1.5 V high speed low-power CMOS current sense amplifier," Electron. Lett., vol. 31, pp. 1991-1993, Nov. 1995.

[12] H. Yamauchi, Y. Iwata, H. Akamatsu, and A. Matsuzawa, "A $0.5 \mathrm{~V}$ single power supply operated high-speed boosted and offset-grounded data storage (BOGS) SRAM cell architecture," IEEE Trans. Very Large Scale Integrated Syst., vol. 5, pp. 377-387, Dec. 1997.

[13] M. Nakamura and N. Ishihara, "1.2 V, $35 \mathrm{~mW}$ CMOS optical transceiver IC's for $50 \mathrm{Mbit} / \mathrm{s}$ burst-mode communication," Electron. Lett., vol. 35, pp. 394-395, Mar. 1999.

[14] T. Kuroda, K. Suzuki, S. Mita, T. Fujita, F. Yamane, F. Sano, A. Chiba, Y. Watanabe, K. Matsuda, T. Maeda, T. Sakurai, and T. Furuyama, "Variable supply-voltagge scheme for low-power high-speed CMOS digital design," IEEE J. Solid-State Circuits, vol. 33, pp. 454-462, Mar. 1998.

[15] M. R. Stan and W. P. Burleson, "Low-power encodings for global communication in CMOS VLSI," IEEE Trans. Very Large Scale Integrated Syst., vol. 5, pp. 444-455, Dec. 1997.

[16] J. T. Ludwig, S. H. Nawab, and A. P. Chandrakasan, "Low-power digital filtering using approximate processing," IEEE J. Solid-State Circuits, vol. 31, pp. 395-400, Mar. 1996.

[17] A. T. Erdogan and T. Arslan, "Low-power coefficient segmentation algorithm for FIR filter implementation," Electron. Lett., vol. 34, pp. 1817-1819, Sept. 1998. 
[18] S. H. Cho, T. Xanthopoulos, and A. P. Chandrakasan, "A low-power variable length decoder for MPEG-2 based on nonuniform fine-grain table partitioning," IEEE Trans. Very Large Scale Integrated Syst., vol. 7, pp. 249-257, June 1999.

[19] C. Deng and C. Chien, "A low energy architecture, for fast PN acquisition," in Proc. ISLPED99, 1999, pp. 42-47.

[20] D. Garrett and M. Stan, "Low power parallel spread-spectrum correlator," Proc. Inst. Elect. Eng. Circuits Devices Syst., vol. 146, pp. 191-196, Aug. 1999.

[21] C.-J. Kim, H. J. Lee, and H.-S. Lee, "Adaptive acquisition of PN sequences for DSSS communications," IEEE Trans. Commun., vol. 46, pp. 993-996, Aug. 1998.

[22] P. Bataille, M. Testard, and G. Levannier, "Performance analysis of noncoherent DPSK receivers for medium data rate EHF frequency-hopping systems-Part 2: Simulation analysis of N-DPSK receivers," in Proc. IEEE Military Communications Conf., vol. 3, Oct. 1996, pp. 1069-1073.

[23] L. M. Arslan and J. H. L. Hansen, "Speech enhancement for crosstalk interference," IEEE Signal Processing Lett., vol. 4, pp. 92-95, Apr. 1997.

[24] B. Ibrahim and A. H. Aghvami, "The performance of a direct sequence spread-spectrum PN code tracking system for frequency selective channels," in Proc. IEEE Int. Symp. Personal, Indoor, Mobile Radio Communications, vol. 3, 1996, pp. 853-857.

[25] S. Dey, A. Raghunathan, and N. K. Jha, "Controller-based power management for control-flow intensive designs," IEEE Trans. Comput._Aided Design Integrated Circuits, Systems, vol. 18, pp. 1496-1508, Oct. 1999.

[26] K. E. Nicholson, R. L. Doughty, L. Mane, G. Miranda, and F. D. Pulaski, "Cost effective power management systems," IEEE Indust. Appl. Mag., vol. 6, pp. 23-33, Mar. 2000

[27] G. Lakshminarayana, A. Raghunathan, N. K. Jha, and S. Dey, "Power management in high-level synthesis," IEEE Trans. Very Large Scale Integrated Syst., vol. 7, pp. 7-15, Mar. 1999.

[28] A. Polydoros and C. L. Weber, "A unified approach to serial search spread-spectrum code acquisition - Part I: General theory," IEEE Trans. Commun., vol. COM-32, pp. 542-549, May 1984.
[29] M.-L. Wu, K.-A. Wen, and C.-S. Chen, "The optimized threshold decision of pseudo noise code acquisition in spread-spectrum communication," IEICE Trans. Fundam. Electron., Commun. Comput. Sci., vol. E83-A, pp. 2152-2159, Nov. 2000.

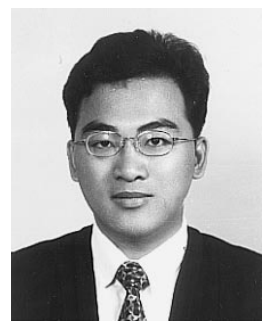

Mau-Lin Wu received the B.E.E. and M.E.E. degrees from the Department of Electrical Engineering and the Institute of Electrical Engineering, National Taiwan University, Taipei, Taiwan, in 1994 and 1996, respectively, and the Ph.D. degree from the Institute of Electronics Engineering, National Chiao Tung University, Hsinchu, Taiwan, in 2001.

From 1996 to 2000 , he was a VLSI Circuit Designer with Taiwan Semiconductor Manufacturing Company, Hsin-Chu, Taiwan. Since 2000, he has been an IC Designer with Integrated Circuit Solution Inc., Hsin-Chu, Taiwan, where he is focused on the design of wireless communication systems.

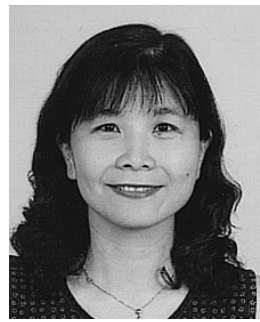

Kuei-Ann Wen received the B.E.E., M.E.E., and $\mathrm{Ph} . \mathrm{D}$. degrees from the Department of Electrical Engineering and the Institute of Electrical and Computer Engineering, National Cheng Kung University, Tainan, Taiwan, in 1983, 1985, and 1988, respectively.

She is currently a Professor with the Department of Electrical Engineering, National Chiao Tung University, Hsinchu, Taiwan. 